\title{
Histoire religieuse du diocèse de Rimouski
}

\section{Léo Bérubé}

Volume 34, 1967

URI : https://id.erudit.org/iderudit/1007423ar

DOI : https://doi.org/10.7202/1007423ar

Aller au sommaire du numéro

Éditeur(s)

Les Éditions Historia Ecclesiæ Catholicæ Canadensis Inc.

ISSN

0318-6172 (imprimé)

1927-7067 (numérique)

Découvrir la revue

Citer cet article

Bérubé, L. (1967). Histoire religieuse du diocèse de Rimouski. Sessions d'étude -

Société canadienne d'histoire de l'Église catholique, 34, 67-74.

https://doi.org/10.7202/1007423ar

Tous droits réservés @ Les Éditions Historia Ecclesiæ Catholicæ Canadensis Inc., 1968
Ce document est protégé par la loi sur le droit d'auteur. L'utilisation des services d'Érudit (y compris la reproduction) est assujettie à sa politique d'utilisation que vous pouvez consulter en ligne.

https://apropos.erudit.org/fr/usagers/politique-dutilisation/ 


\section{Histoire religieuse du diocèse de Rimouski}

L'histoire ecclésiastique canadienne serait bien incomplète si elle omettait l'histoire de l'Eglise du Bas Saint-Laurent. Elle s'amputerait d'une réalité dont la naissance tient de l'héroïsme et dont la fécondité est toujours nouvelle. Retracer la geste glorieuse de notre Eglise territoriale, c'est évoquer les interminables voyages de nos premiers missionnaires; c'est aussi faire renaître la figure de nos évêques, de nos prêtres et de notre peuple tout entier. "Peuple, dit le chanoine Groulx, qui a lié, rivé sa vie à l'Eglise, à cette puissance d'éternelle jeunesse, de résurrections toujours possibles et prochaines ${ }^{1}$.

Dans son premier siècle, l'histoire religieuse de notre région se confond avec l'histoire canadienne-française, celle-ci pouvant se définir en deux mots : climat d'apostolat.

Les croix plantées par Cartier et Champlain révèlent leur signification, trouvent leur efficacité dans la célébration du saint Sacrifice de la Messe : sacrifice célébré un peu partout, au hasard des haltes des missionnaires ou des mouillages de bateaux, et qui ensemence notre sol pour une moisson d'authentique christianisme.

Dès le temps de Cartier, la Messe avait été dite "sur la côte du Labrador, à l'Ile-aux-Coudres, sur les bords de la rivière Saint-Charles, au Cap-Rouge, peut-être aussi à Hochelaga ${ }^{2}$ ".

Il faut attendre l'année 1615 pour retrouver cet événement. Les Récollets sont alors au pays et ils se répartissent la tâche de la conversion des peuplades de l'Amérique du Nord. Le père Dolbeau reçoit en partage l'évangélisation « des Montagnais du Saguenay et de la partie inférieure du Saint-Laurent ${ }^{3}$ ", depuis Tadoussac jusqu'à Betsiamits et même plus loin que Sept-Iles.

Le bateau qui ramena les missionnaires de France, en 1632, mouilla à Gaspé; des pères jésuites y célèbrèrent la messe le 13 juin, et à Tadoussac, le 18 juin. Arrivés à Québec le 5 juillet, ils regagnèrent immédiatement les forêts et les rares postes où les attendaient Français et Indiens. Pour mieux apprendre l'idiome montagnais, le père Paul Le Jeune * s'astreint à passer tout son premier hiver dans le bois, en arrière de Rimouski, l'Isle-Verte, la Rivière-du-Loup ${ }^{4}$ ».

A partir de 1635, les Jésuites ont une résidence " à l'île Miscou, à l'entrée de la baie des Chaleurs ${ }^{5}$, où l'on signale le passage du père

1 L. Groulx, Notre Maître, le Passé, Granger, 1944, p. 231.

p. 17 .

A. Gosseur, La mission du Canada avant Mgr de Laval, Evreux, 1909,

Ibid., p. 20

4 Ibid., p. 59.

5 Relations des Jésuites pour l'année 1635, p. 3. 
Sébastien, récollet, en 1620. Pendant plusieurs années, les Indiens de la baie et de la côte gaspésienne donnèrent à ces missionnaires de grandes consolations.

Du côté de Québec, ce sont les pages glorieuses des Martyrs Canadiens qui s'écrivent à cette époque. Pendant un siècle, donc, le sang du Christ et le sang de ses martyrs préparèrent la fondation officielle de l"Eglise du Canada.

Le 11 avril 1658, à la demande des Jésuites, Mgr François de Montmorency Laval est nommé vicaire apostolique en terre de NouvelleFrance. Son diocèse futur ne comprend pas moins que atoutes les possessions françaises de l'Amérique du Nord, depuis les bouches du Mississipi aux Grands-Lacs, et des Grands-Lacs à la mer, jusqu'à l'Acadie et Terre-Neuve ${ }^{6}$ ».

Les Relations des Jésuites, pour l'année 1664, rapportent le détail d'un voyage sur la côte du Saint-Laurent. Le héros de ce voyage n'est autre que le père Henri Nouvel, parti de Québec le 19 novembre 1663, faisant escale forcée à l'île Verte, puis abordant vis-à-vis de l'île SaintBarnabé où, le 8 décembre, il célèbre la messe. Le nom de Pointe-auPère immortalise cet événement.

Et selon la chronologie, c'est encore le père Albanel qui passe l'hiver à la Rivière-du-Loup et dans les îles environnantes, en 1668, et le père François de Crépieul qui se dévoue, de 1671 à 1702, au service des Blancs et à la conversion des Indigènes dans toute la Gaspésie, au Saguenay, jusqu'en forêt labradorienne.

Puis, l'ère des seigneuries commence dans notre région. Elles seront concédées d'abord pour la pêche, mais la seigneurie de Rimouski, celle des Trois-Pistoles et celle de l'Isle-Verte auront bientôt l'avantage d'appartenir à de vrais colonisateurs. L'arrivée des seigneurs René Lepage et Jean Rioux, en 1696, et du seigneur Jean-Baptiste Côté, en 1711, marque en effet l'ouverture du diocèse de Rimouski à la colonisation. Elle marque aussi le point de départ de son organisation religieuse, car l'Eglise suit ses enfants et les colons ne sauraient se passer d'elle.

Tout nouveau poste a sa petite chapelle. Ce sont les missionnaires de passage, en l'occurrence les Récollets, qui y exercent d'abord les fonctions du culte. Le père Bernardin Leneuf, qui s'arrête à Rimouski en 1701, procède à l'ouverture du plus ancien registre paroissial que nous ayons au diocèse.

Les fils de saint François d'Assise continueront de desservir, occasionnellement d'abord, puis presque régulièrement ensuite, les établissements seigneuriaux de cette partie de la côte jusqu'en 1767. Quand ils devront prolonger leur absence, les curés de Kamouraska seront mandatés pour les remplacer.

Pendant ces décennies, dans la lointaine Gaspésie, on est en butte à une difficulté nouvelle : la menace anglaise. Les Récollets, qui avaient

6 L. Groulx, Histoire du Canada français, t. 1, Fides, 1960, 394 p. 166. 
pris la relève des Jésuites et qui s'étaient "établis à demeure à Percé en $1673^{7}$, sont les innocentes victimes de l'invasion de 1690. Leurs missions sont saccagées et ils se voient contraints, pour la plupart, de retourner à Québec. A Gaspé, comme dans le reste de l'Acadie, la vie perd de son élan et se charge d'inquiétude; c'est à se demander si l'acharnement de l'envahisseur n'aura pas raison de tant de germes de foi déposés dans les âmes.

Mais les années passent et Dieu permet une fois de plus que le bien soit tiré du mal. En effet, le peuple qui a le plus à souffrir de la cruauté des Anglais, le peuple acadien, est celui-là même qui vient ensuite régénérer la Gaspésie, l'ouvrir définitivement à la colonisation et en faire un véritable foyer de vie religieuse.

Certes, les Acadiens fugitifs de 1755 ne sont pas seuls en terre gaspésienne : ils ont avec eux, surtout à Carleton et à Bonaventure, pour ne parler que de leurs débuts, des chefs de file qui sont encore les missionnaires. Nous ferons spécialement mention du père Bonaventure Charpentier qui fut leur soutien, leur consolation et leur guide à l'époque du grand Dérangement, et de l'abbé Mathurin Bourg, un compatriote, qui se fit surtout leur défenseur contre les empiètements les plus audacieux des Loyalistes.

Ainsi donc, la région mitoyenne du Bas Saint-Laurent et la Gaspésie commencent à peu près en même temps à recevoir des colons et à créer des postes. Mais avec quelle lenteur elles procèdent l'une et l'autre à la multiplication des paroisses ! Après plus d'un siècle, soit à la fondation du diocèse de Rimouski, celles-ci ne seront encore qu'au nombre de 32 .

C'est que ce coin de pays est proprement victime de son isolement ${ }^{8}$. Il est loin des pouvoirs publics qui devraient l'aider dans son développement agricole, lui construire des routes et lui faciliter l'exploitation de la pêche, sa principale industrie; loin aussi de l'Eglise-mère de Québec qui s'attarde trop peut-être, à cause de ses besoins multiples et du manque de prêtres, à le considérer comme une terre de missions.

Pour peu qu'il se sente abandonné, un peuple se laisse facilement subjuguer par les puissances aventureuses qui lui promettent l'assistance immédiate. Or, en Gaspésie particulièrement, il s'est trouvé que des exploiteurs étrangers ont abusé de la situation en maintenant longtemps dans une odieuse servitude des gens qui n'aspiraient qu'à vivre convenablement, à prospérer, à enrichir le domaine de l'Etat et à propager l'influence de l'Eglise.

A l'heure où il aurait fallu que le clergé usât d'autorité et de zèle pour refréner un tel désordre, les prêtres étaient en trop petit nombre. En 1785, il n'y en avait que deux dans tout le territoire qui s'étend de la Rivière-du-Loup à Gaspé : un abbé Leclerc à l'Isle-Verte et l'abbé Bourg à Carleton. Trente-cinq ans plus tard, soit en 1820, ils ne seront encore que quatre dans le même territoire.

7 A. Bernard, La Gaspésie au Soleil, p. 121.

8 Ibid., p. 201. 
Mais la région du Saint-Laurent inférieur commence alors à sortir de sa léthargie. Elle attire de plus en plus les agriculteurs des vieilles paroisses de la rive sud, et avec eux, quelques maîtres de l'industrie forestière. C'est encore un pays tout nouveau, mais il se peuple à un rythme accéléré, il s'anime, et d'année en année, il multiplie ses postes.

Les évêques de Québec suivent de près cette évolution. S'ils manquent toujours douloureusement de prêtres, ils ne sont pas moins disposés à faire de louables efforts pour mettre les secours spirituels le plus

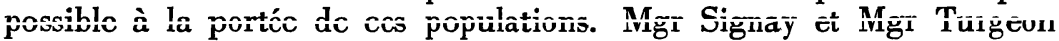
font preuve de beaucoup de sollicitude, mais les résultats apparaissent surtout sous Mgr Baillargeon.

On voit ce prélat, qui prend la direction du diocèse en 1855 , porter de six à quatorze le nombre des prêtres de la Gaspésie, placer des curés dans les postes nouveaux qui ont surgi sur le littoral, entre Cacouna et Matane, et pourvoir de missionnaires la région du Témiscouata et la Côte Nord. De plus, il favorise la fondation de couvents de religieuses enseignantes à Rimouski, à Cacouna, aux Trois-Pistoles et à Carleton, et il assure le fonctionnement du collège classique, le futur Séminaire de Rimouski, mis sur pied en 1862.

Il est évident qu'un plan bien arrêté mijote dans l'esprit de Mgr Baillargeon : organiser au plus tôt cette partie reculée du diocèse de Québec pour lui faire donner son autonomie religieuse.

L'idée d'un nouveau diocèse n'est pas particulière à l'évêque; elle fait son chemin et la préparation est toujours plus active. On se perd d'abord en conjectures sur le choix du chef-lieu, mais en juillet 1866 , les évêques, qui sont réunis à Saint-Hyacinthe, arrêtent leur préférence sur Rimouski, en pensant à bon droit qu'une nouvelle division pourrait en détacher plus tard la Gaspésie proprement dite.

Le fruit a mûri rapidement. Le 15 janvier 1867, Pie IX érige en diocèse, sous le vocable de Saint-Germain de Rimouski, les districts de Rimouski et de Gaspé, le comté de Témiscouata, moins les paroisses de Saint-Patrice de Rivière-de-Loup, de Saint-Antonin et de Notre-Dame du Portage, et sur la rive nord, tout le territoire compris entre la rivière Portneuf et l'anse de Blanc-Sablon. Ces limites contiennent 60,000 âmes.

A la nouvelle église, le Pape donne pour chef un prêtre de choix rompu à toutes les besognes, à l'enseignement, à la direction des groupes, à l'administration paroissiale, l'abbé Jean Langevin, principal de l'Ecole Normale Laval, qui est sacré évêque le premier mai suivant. Le jeune pasteur s'amène sur les lieux aussitôt que la navigation le permet, le 17 mai, et le voici en état d'embrasser du regard l'étendue de sa tâche.

C'est un champ en friche que le sien. Il aura à le remuer, à l'ameublir et à l'amender pour lui faire produire la moisson dorée que sa charité ne peut que souhaiter et que son zèle entrevoit déjà. Mais comme il sent le besoin du secours d'en haut pour accomplir son ouvre! La 
première prière qu'il fait dans sa cathédrale en est une sans doute de sollicitation.

Certes, Mgr Langevin sait découvrir que la machine est déjà en marche et qu'il y a ici et là des réalisations heureuses. Mais que ne reste-t-il pas à faire pour fondre en une seule famille ces régions disparates, pour leur inculquer un esprit diocésain!

S'il fait le compte de son clergé, il trouve 46 prêtres. Il en faudrait bien davantage pour assurer le succès des œuvres déjà commencées, pour créer les principaux organismes de fonctionnement, pour pourvoir les centres nouveaux, pour faire prospérer le collège et pour alléger quelque peu le fardeau de certains missionnaires.

De même, s'il procède au bilan de ses ressources, il constate qu'elles sont nettement insuffisantes. Car c'est un diocèse pauvre qu'il entreprend d'organiser. Deux des circonscriptions surtout. la Côte Nord et la Gaspésie, n'ont pas du tout ou que très peu de développement. Quant au district de Rimouski et au comté de Témiscouata, ils comptent un plus grand nombre d'établissements agricoles, mais ils sont généralement jeunes encore et peu prospères.

Enfin, s'il s'arrête à regarder sa situation, il se voit dans une résidence empruntée, dans un simple village de campagne, en plein pays de colonisation, à plus de soixante milles du chemin de fer, loin de tous les grands centres et privé de bien des facilités auxquelles il était habitué. Mais rien de tout cela ne peut l'ébranler. C'est en homme de foi qu'il s'attelle à la tâche et il semble trouver rapidement les orientations à donner à son action.

On le constate aujourd'hui : le premier évêque de Rimouski a tracé, dès le début de son ministère épiscopal, des plans d'apostolat que ses quatre premiers successeurs ont travaillé comme lui à réaliser : entretien et vitalité du séminaire diocésain; promotion de l'éducation et de l'instruction; travail acharné en faveur de la colonisation et de l'agriculture; lutte pour la stabilité des diocésains, pour la tempérance et la modestie; soutien de la vie chrétienne par la prédication aux adultes, le catéchisme aux enfants, les confréries de toutes sortes; organisation de la vie familiale et de la vie paroissiale; appels incessants à la pratique des vertus sociales, au respect de l'autorité, etc.

Parce que tout est à faire, tout à organiser, tout à ordonner, Mgr Langevin est sans cesse dans l'occasion d'exercer à la fois ses rares talents d'éducateur, de pasteur et d'administrateur. On le voit instituer une discipline diocésaine, créer des organismes d'administration, construire un évêché et un séminaire, relever le niveau de l'enseignement primaire, assurer le recrutement du clergé, introduire des œuvres de bienfaisance, établir des sociétés de colonisation et d'agriculture et fonder des paroisses.

Nous pourrions multiplier à loisir les preuves de sa sollicitude pour tous les aspects de la vie de ses ouailles. Il veut évidemment pour elles la sécurité matérielle promise à l'attachement au sol, il veut l'accès 
à la culture intellectuelle, une vie patriotique authentique et une vie chrétienne fervente. D'un peuple trop engourdi par différents facteurs sociaux, il veut faire naître une famille unie dans la liberté véritable et dans la noblesse.

L'ouvrier s'est usé à la tâche, cela va sans dire, car il s'est dépensé à la façon de l'Apôtre ${ }^{9}$, il s'est donné tout entier. Mais il n'aura pas travaillé en vain. Dans les vingt-quatre années de son épiscopat. Mgr Langevin a posé le diocèse sur des bases solides et lui a fait prendre

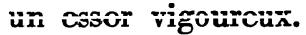

Il avait commencé avec 60,000 âmes; il termine avec 90,000 , même si la Côte Nord, depuis 1882, ne fait plus partie de Rimouski. Le nombre des paroisses organisées est passé de 32 à 70 et celui des prêtres, de 46 à 92. Les écoles paroissiales sont au moins quatre fois plus nombreuses et surtout, elles peuvent compter sur la compétence et le zèle des Sœurs des Petites-Ecoles, que l'évêque a fondées en 1874, et qui sont devenues, sous son successeur, la florissante Congrégation de Notre-Dame du Saint-Rosaire. Le territoire lui-même, malgré son démembrement, a presque triplé son domaine agricole, et il n'est plus question pour luj $\mathrm{d}$ 'isolement, car le chemin de fer le traverse et le relie au reste du pays.

Et que dire de la valeur de l'Eglise de Rimouski au départ de son fondateur? Certes, elle n'est pas encore à l'apogée de son développement, mais que de progrès elle a déjà réalisés ! Là où l'indifférence avait fini par s'implanter, une ferveur commune animait les esprits. On était plus fidèle à ses devoirs de chrétien, plus soucieux de la pratique des vertus et plus attaché aux choses de la religion. Cela tient à ce que le peuple était plus instruit de ses obligations et le clergé, plus conscient de ses devoirs.

Le 30 décembre 1889, Rome donnait à Mgr Langevin, comme coadjuteur, l'abbé André-Albert Blais, de Québec. Il reçoit la consécration épiscopale le 18 mai 1890 et il prend le titre d'évêque de Rimouski le 6 février 1891. C'est l'homme à l'éloquence facile et abondante, qui se fait remarquer par sa grande distinction et l'urbanité de ses manières; c'est aussi le savant, le travailleur et le diplomate.

La jeune Eglise de Rimouski a alors besoin d'un tel chef pour consolider ses positions, perfectionner ses institutions et s'élever au niveau de ses sœurs, les autres chrétientés canadiennes.

Effectivement, elle complète son organisation et elle grandit. On passe de la colonisation à l'agriculture et de l'habitation provisoire à la demeure définitive. Nos églises de pierre, par exemple, sont. pour la plupart, de cette époque. Le progrès ne touche pas moins les domaines du bien-être, de la santé, de l'éducation, des relations sociales. voire de la piété.

Après les vingt-huit années que dure l'épiscopat de Mgr Blais, le diocèse, qui est devenu quinquagénaire, a pris un tel développement que

911 Cor. XII, 15. 
la question se pose de le diviser. Il compte 96 paroisses formellement érigées, 11 dessertes, 24 missions, en tout 131 clochers. Il y a 170 prêtres, une quinzaine de communautés religieuses différentes et une population de 142,000 âmes.

Cette division du diocèse s'opère en effet sous le troisième évêque, Mgr Joseph-Romuald Léonard, en 1922. Ce sont 52,000 fidèles, environ 50 prêtres et une soixantaine de centres religieux qui s'en détachent pour le nouveau diocèse de Gaspé. Mais nous sommes au lendemain de la Première Grande Guerre, à l'époque où la propagande agricole bat son plein, et le vide ne tarde pas à se combler.

Le frêle Mgr Léonard, un fils de la Gaspésie, n'est pratiquement à la tâche que pendant six ans, de 1920 à 1926 . Une si brève administration ne saurait se comparer aux longs règnes de ses prédécesseurs; elle a cependant produit des œuvres aussi durables que précieuses et surtout, elle a marqué la vie diocésaine du triple caractère de l'ordre, de la discipline et de la ferveur religieuse.

En 1928, l'Eglise de Rimouski accueille son quatrième évêque, Mgr Georges Courchesne. Cet éminent Nicolétain est précédé ici par sa réputation de grand intellectuel, de penseur, même de spécialiste des questions sociales. Il se réserve toutefois de nous apprendre lui-même que, « fils de cultivateur, il a gardé pour le peuple agriculteur une sympathie attendrie ${ }^{10} \%$. Ce sentiment intime, il ne l'écrit pas seulement: il le vit intensivement pendant les vingt-deux années de son épiscopat.

Il faut dire que les circonstances s'y prêtaient à merveille. En effet, la gestion de Mgr Courchesne s'est édifiée en bonne partie sur les ravages causés par la trop célèbre crise économique qui a précédé la Seconde Grande Guerre. Obligé de lutter contre le chômage généralisé et désireux de retenir en place le capital humain du diocèse, il s'est fait en quelque sorte le Curé Labelle de son époque, pour voler à la forêt rimouskoise les dernières parcelles de son sol cultivable et garder à l'agriculture sa légitime considération.

Le vaillant évêque ne s'est pas moins révélé le défenseur vigilant et l'animateur de la vie de l'esprit. Il suivait partout ses diocésains et, à quelque rang qu'ils appartenaient, il les entraînait presque malgré eux dans le champ de la réflexion et de l'étude, afin de les ancrer dans le respect et l'amour de leur profession. Pour cela, il a multiplié et perfectionné les œuvres d'éducation et doté la société, qui évoluait déjà rapidement, d'organismes nouveaux plus conformes à ses besoins.

En étendant sa zone d'habitation, en renouvelant et complétant son organisation sociale et éducationnelle et en augmentant considérablement sa population, le diocèse avait acquis de l'importance. Il n'y a donc pas lieu de s'étonner de le voir s'élever au rang d'église métropolitaine en 1946. Mgr Courchesne devenait le premier archevêque de Rimouski.

10 G. Courchesne, Mandements et Circulaires, Vol. 1, p. 3. 
Le Saint-Siège désigna pour lui succéder, en 1951, celui-là même qu'Il lui avait donné comme auxiliaire en 1944, Mgr Charles-Eugène Parent. Pour la première fois, le pouvoir ecclésiastique de Rimouski est entre les mains d'un authentique Rimouskois.

La vie diocésaine ne change guère d'abord sous son pastorat, si ce n'est que la plupart des œuvres déjà établies reçoivent une impulsion nouvelle qui les rend plus efficaces, et une attention qui leur permet d'être encore mieux adaptées aux nécessités du temps.

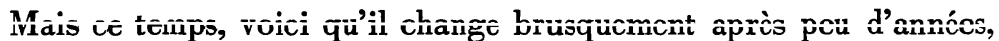
à l'annonce d'un concile ocuménique. Vatican II vient en effet remuer tous les hommes. Il s'agit d'abord de le préparer, et c'est par la prière, l'étude de la Bible, une meilleure compréhension de la liturgie et surtout, le soin de vivre déjà avec plus de vigueur toutes les exigences du christianisme. Il s'agit ensuite d'appliquer le Concile, et c'est toute une réorganisation de la vie de l'Eglise qui commence dans le diocèse. Aux plans d'action individuelle, se substitue graduellement le mouvement d'ensemble. Les œuvres deviennent des activités de collaboration où prêtres, religieux, religieuses et laïques forment équipe pour le plus grand bien du Peuple de Dieu.

Les réalisations de l'Eglise diocésaine pour cette période qui va de 1951 à 1967, elles sont sous les yeux de tous. Il serait vain de les vouloir décrire ou raconter. Donnons plutôt quelques statistiques.

Le diocèse de Rimouski compte aujourd'hui 120 paroisses et une population catholique de 176,000 âmes. Nous trouvons à son service 360 prêtres, dont 296 appartiennent au clergé séculier et 64 , à six familles religieuses différentes. Dix-sept congrégations de Sœurs et quatre de Frères, groupant en tout quelque 1300 membres, sont à l'œuvre dans l'enseignement et les institutions de bienfaisance.

Le diocèse a aussi son actif missionnaire. De nombreux apôtres le représentent depuis longtemps dans les pays où l'évangélisation est toujours affaire de dévouement et de sacrifices de toutes sortes. Récemment encore, il a mis trois de ses prêtres à la disposition du Brésil.

Voilà donc comment l'Eglise de Rimouski a vécu son premier siècle. Ce fut vraiment un siècle riche de figures éminentes, d'œuvres remarquables et de développements intensifs dans presque toutes les sphères de l'activité humaine.

Au moment même où cette Eglise s'apprêtait à célébrer son centenaire, l'hiver dernier, elle était confiée à la sollicitude pastorale d'un nouveau chef, Mgr Louis Levesque, un autre fils du diocèse. Il lui a déjà imprimé la vigueur et le dynamisme qui avaient caractérisé l'Evêque de Hearst. C'est maintenant une institution qui a nettoyé son phare, hissé plus solidement sa voile et invité tous ses membres à une action communautaire.

Ce qui lui fut gracieusement apporté par les hérauts de l'Evangile, la foi, l'espérance et l'amour, elle veut continuer de le répandre avec profusion dans toutes les âmes de bonne volonté. Toute l'Eglise du Christ peut compter sur elle.

Chan. Léo BÉRUBÉ 